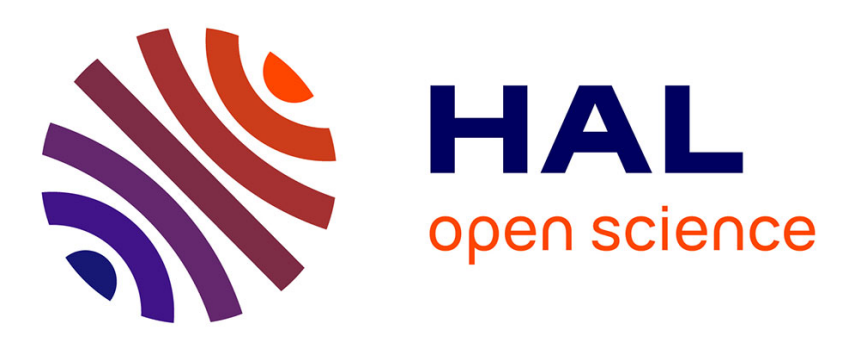

\title{
Des Indiens dans les armées hellénistiques: une autre rencontre entre la Grèce et l'Inde
}

Pierre Schneider

\section{To cite this version:}

Pierre Schneider. Des Indiens dans les armées hellénistiques: une autre rencontre entre la Grèce et l'Inde. Archimède: archéologie et histoire ancienne, 2017, 4, pp.229-235. halshs-01589457

\section{HAL Id: halshs-01589457 https://shs.hal.science/halshs-01589457}

Submitted on 18 Sep 2017

HAL is a multi-disciplinary open access archive for the deposit and dissemination of scientific research documents, whether they are published or not. The documents may come from teaching and research institutions in France or abroad, or from public or private research centers.
L'archive ouverte pluridisciplinaire HAL, est destinée au dépôt et à la diffusion de documents scientifiques de niveau recherche, publiés ou non, émanant des établissements d'enseignement et de recherche français ou étrangers, des laboratoires publics ou privés. 


\section{ARCHIMÈDE N4}

\section{DOSSIER THÉMATIQUE 1 : NOMMER LES « ORIENTAUX » DANS L’ANTIQUITÉ}

\section{DOSSIER THÉMATIQUE 2 : PRYTANÉE ET REGIA}

\section{ACTUALITÉ DE LA RECHERCHE : DYNAMIQUES HUMAINES ANCIENNES}

155 Steeve GENTNER, Thomas HUTIN et Šárka VÁLEČKOVÁ

Introduction au dossier : les phénomènes de hiérarchisation et leurs traitements dans les sociétés anciennes et actuelles

158 Pierre LE ROUX et Alain BEYRAND

D'un drame à un don. Hommage à Louise Beyrand et Olivier Toussaint

162 Alexandra CONY

Hiérarchie de I'habitat rural à la fin de l'âge du Fer. L'importance de définir les critères de hiérarchisation en fonction de l'environnement archéologique

174 Christine HUE-ARCÉ

Hiérarchies socio-professionnelles et violence interpersonnelle dans l'Égypte du Nouvel Empire et d'époque hellénistique

184 Aurélien LANDON

La hiérarchie dans le recrutement militaire : I'exemple épigraphique du recrutement des tribuns militaires durant le Principat d'Auguste

196 Sophie TRIERWEILER

La conception et l'expression d'un droit structuré dans la société homérique à travers les notions de themis et dikê

207 François FAVORY

Organisation et hiérarchisation de l'habitat antique : I'expérience d'Archaeomedes et d'ArchaeDyn

VARIA

216 Jean-Claude LACAM

Prestota Serfia Serfer Martier, la déesse immobile (étude ombrienne, $\mathrm{III}^{\mathrm{e}}-\mathrm{II}^{\mathrm{e}}$ siècles av. J.-C.)

\section{LA CHRONIQUE D’ARCHIMÈDE}

236 Frédéric COLIN (éd.)

La Chronique d'Archimède. Bilan des activités scientifiques 2016-2017 de l'unité mixte

\section{@creative $(1) @(1)$}




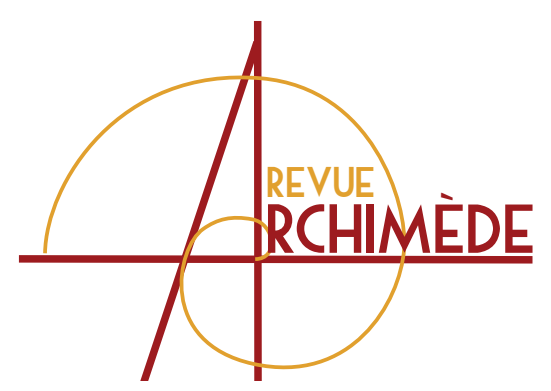

ARCHÉOLOGIE ET HISTOIRE ANCIENNE

\section{DES INDIENS DANS LES ARMÉES HELLÉNISTIQUES : UNE AUTRE RENCONTRE ENTRE LA GRÈCE ET L'INDE}

Pierre SCHNEIDER

Maître de conférences HDR en histoire ancienne

Université d'Artois

EA 4027 CREHS \& UMR 5189 Hisoma

pierre.schneider@univ-artois.fr

\section{RÉSUMÉ}

L'un des traits majeurs de l'époque hellénistique est I'intensité des contacts culturels entre les Hellènes et les peuples non-grecs. Une partie de ces contacts ont eu pour cadre les armées royales. Longtemps il s'y est trouvé des Indiens, souvent des cornacs. Certains étaient accompagnés de leurs épouses, de leurs enfants et de leur famille. Cet article passe en revue les traces documentaires de ces contacts culturels entre Hellènes et rencontres culturelles. Indiens et l'impact qu'ils ont pu avoir sur l'image de I'Inde dans le monde méditerranéen.
The numerous cultural contacts that Greeks had with non-Greek peoples characterize the Hellenistic period. Royal armies, due to their multicultural composition, were particularly suited for such contacts. This paper focuses on the Indians enrolled in Hellenistic armies, mainly as mahouts, some of them being accompanied by their wives, children and parents. A couple of ancient texts give an idea of what kind of cultural contacts occured in such an environment. It is also argued that these impacted (to some extent) on the representation of India that prevailed in the Mediterranean world.
Keywords Ancient India, Hellenistic armies, cross-cultural contacts. 
Un volume des Dialogues d'histoire ancienne avait, naguère, été consacré aux rencontres entre la Grèce et I'Inde [1]. Les contributeurs, hellénistes ou indianistes, avaient exploré les diverses formes d'interpénétrations, d'influences ou d'analogies - il est difficile de s'accorder sur un terme satisfaisant entre ces deux mondes. Il en ressort que l'étude des rencontres culturelles entre la Grèce et I'Inde, de I'expédition d'Alexandre jusqu'à la fin de l'Antiquité, se concentre sur la sphère des savoirs lettrés - par exemple, les contacts incarnés par Empédocle, Onésicrite, Kalanos, Apollonios de Tyane etc. - et celle des savoirs pratiques - par exemple, les remèdes et la médecine- ; il faut y ajouter les domaines politique et diplomatique - on pense, par exemple, aux ambassades séleucides et indiennes, telle celle relatée dans un décret d'Açoka [2]. L'on songe moins aux contacts qui purent s'établir en dehors des cercles savants et des cours royales, en particulier au sein des armées de la haute époque hellénistique. C'est dans ce cadre, en effet, que des Hellènes et des Indiens, des gens ordinaires pour la plupart, s'y côtoyèrent, non seulement en Asie, mais aussi en Europe. Quelques documents, aussi rares qu'inattendus, restituent les traces de ces contacts passés. Et, comme on le verra, ces rencontres obscures et anonymes contribuèrent - modestement mais indiscutablement - à façonner l'image que le monde grec se fit de I'Inde.

\section{UN INDIEN ET SA FAMILLE DEVANT MÉGARE (272 A. C. ?)}

La disparition d'une partie de la Bibliothèque Historique de Diodore de Sicile rend difficile I'établissement de certains faits d'histoire politique du

\section{[1] Muckensturm-Poulle \& Geny 2010.}

[2] Sur les relations entre le monde méditerranéen et l'Inde voir les articles et ouvrages suivants (liste non exhaustive) : RaWLinson 1926 ; Filliozat 1956 ; Treloar 1977 ; Sedlar 1980 ; Doshi 1985; Bongard-Levin 19851986. On y trouvera toutes les références se rapportant aux personnages et faits évoqués ci-dessus.

[3] Justin, Prologue, 26.

[4] Stobée, Peri Xenes, XL 8.

[5] Il s'agit des cornacs et de tous ceux qui nourrissaient, soignaient et entraînaient les éléphants.

[6] Polyen, Stratagèmes, IV 6, 3. Une autre version de monde hellénistique au $\mathrm{III}^{\mathrm{e}}$ siècle $a$. C. On compte au nombre de ceux-ci le siège de Mégare qu'avait mené Antigonos Gonatas. On en ignore presque tout, tant le dossier documentaire est maigre. Il se réduit principalement à un prologue de TroguePompée [3], un passage de Stobée [4] et enfin à quelques lignes de Polyen décrivant comment les Mégariens firent reculer les éléphants de combat — indiens - de l'armée de Gonatas :

«Alors qu'Antigonos assiégeait Mégare, il fit avancer ses éléphants. Les Mégariens lâchèrent des porcs qu'ils avaient enduits de poix liquide et auxquels ils avaient mis le feu. Ceux-ci, que les flammes consumaient, poussaient des cris perçants et, dans leur course effrénée, ils foncèrent vers les éléphants. Ces derniers, sombrant dans la panique et le désordre, s'enfuirent dans tous les sens. Antigonos ordonna aux Indiens [5] de désormais élever des porcs à côté des éléphants, afin que les pachydermes s'habituent

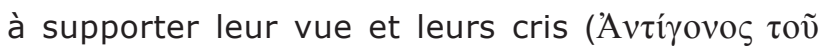

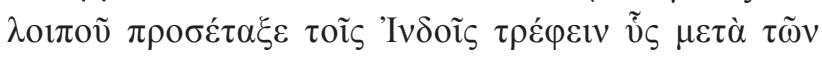

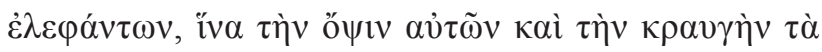

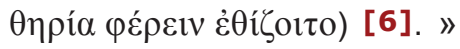

La date et les circonstances du siège de Mégare sont mal établies. Les historiens qui se sont penchés sur la question le situent entre la mort de Pyrrhos (272 a. C.) et le début de la guerre chrémonidéenne (268 a. C.) [7]. H. Heinen n'excluait pas une datation plus haute, entre 277 et 274 a. C. [8] Ce segment chronologique avait été écarté, en son temps, par W. W. Tarn, accordant une importance particulière à la présence d'éléphants dans l'armée de Gonatas. L'événement se serait passé entre 272 et 270, puisque c'est seulement après la mort de Pyrrhos que le roi des Macédoniens aurait récupéré ses éléphants [9].

I'histoire est donnée par Élien, De la nature des animaux, N. A., XVI, 36. Les éléphants appartiennent soit disant à l'armée d'Antipatros, mais on a démontré depuis longtemps qu'il ne peut s'agir que d'Antigonos Gonatas (LE BAS 1842).

[7] Voir, par exemple, HAMmOND 1988, p. 281-282.

[8] HeINEN 1972, p. 170-172.

[9] TARN 1913, p. 286, n. 29 : « Therefore the war with Pyrrhos is also excluded. It must then fall here, where I have placed it, or in the Chremonidean war. The fact that Megara does not appear among Sparta's allies in Syll. 214 is by no mean conclusive against the latter date : Megara may have joined later or been trying to preserve neutrality. » 
Antigonos Gonatas n'était pas le premier monarque, ou presque monarque d'Europe à posséder des éléphants de guerre indiens - cependant que la domestication des éléphants africains commençait tout juste, à Carthage et dans le royaume lagide [10] - : Antipatros, Polyperchon, Cassandros, et Pyrrhos, pour ne citer que ceux-ci, l'avaient précédé. Quant à la présence d'éléphants au siège de Mégare, elle est confirmée par une autre anecdote, figurant chez Élien :

« À l'époque où Antigonos assiégeait les Mégariens, un des éléphants de combat était accompagné d'une femelle, appelée Nikaia. Or l'épouse de son cornac ( $\dot{\eta}$

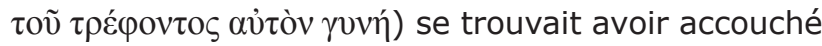
trente jours plus tôt. Elle avait confié à celle-ci son nourrisson en faisant usage de la langue indienne,

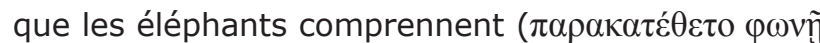

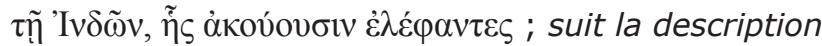
des gestes d'affection et de protection maternelles que l'éléphante avait pour le bébé) [11]. "

On trouve chez I'historien Phylarque, cité par Athénée de Naucratis, une version parallèle de cette anecdote [12]. Elle apparaissait dans les Historiai, qui devaient suivre de quelques décennies les événements. Le texte d'Athénée omet de signaler le siège de Mégare mais fournit quelques détails qui manquent au récit d'Élien au sujet de Nikaia, véritable mère de substitution : l'épouse du cornac

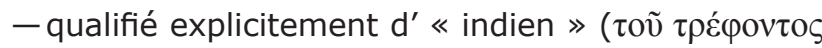
'Iv $\delta$ oṽ) - , à l'agonie trente jours après son accouchement, avait confié son bébé à l'éléphante, laquelle fit preuve d'une incroyable tendresse à son égard

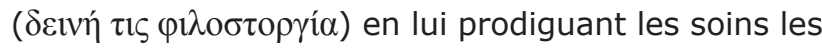
plus attentionnés. En revanche, la compréhension innée de la langue indienne n'est pas signalée.

Il est difficile de déterminer si cette éléphante et ses congénères de l'armée de Gonatas étaient les survivants de ceux qu'Alexandre le Grand avait ramenés de I'Inde à Babylone. Si I'on suit le raisonnement de W. W. Tarn, le siège de Mégare se déroule après 272 , c'est-à-dire cinquante ans après la mort
d'Alexandre. Les éléphants, âgés d'au moins cinquante-cinq, seraient alors assez vieux. Il se pourrait qu'à la faveur de bonnes relations entre Gonatas et Antiochos Ier, le premier ait obtenu du second des éléphants indiens plus jeunes [13]. Quoi qu'il en soit, ceux-ci, capturés et dressés en Inde, étaient accompagnés du personnel indien indispensable pour les conduire et les soigner. Par ailleurs, indépendamment de la question de l'âge des éléphants d'Antigonos, il est plus que vraisemblable que l'épouse du cornac indien, étant assez jeune pour donner naissance à un bébé, était arrivée dans le monde méditerranéen après la disparition d'Alexandre. Bref, nous savons qu'aux alentours de 270, l'armée de Gonatas, disposant d'éléphants indiens, comprenait dans ses effectifs des cornacs indiens - tout comme, précédemment, les armées d'Antipatros, Pyrrhos, Ptolémée Keraunos etc. Ces Indiens, ou certains d'entre eux, étaient accompagnés d'une ou plusieurs épouses (voir ci-après), voire de leur famille. De plus, les soins à prodiguer aux éléphants de combat nécessitaient sans doute la présence d'autres hommes - des Indiens encore ? - assistant les cornacs ou les remplaçant en cas de blessure ou de mort au combat : les cornacs étaient, en effet, la cible des projectiles ennemis. Et quand bien même le chef du bataillon des éléphants pouvait être un Grec [14], les conducteurs, héritiers d'une tradition millénaire dans leur contrée d'origine, ne l'étaient pas, de toute évidence [15].

L'historien peut donc se réjouir que Phylarque ait conservé cette anecdote pathétique - d'un style qui lui avait valu d'être critiqué dans l'Antiquité [16]. Elle prouve que, durant ces longues années de guerre qui suivirent la mort d'Alexandre, des Indiens - cornacs et autres hommes de l'art - avaient circulé en Méditerranée orientale, Grèce d'Europe comprise. Ils faisaient, à n'en pas douter, usage de leur langue et avaient conservé leurs coutumes (nomima), même si certains devaient avoir une connaissance minimale du grec pour agir sur le champ de bataille.
[10] Sur les éléphants de Carthage et ceux des Ptolémées voir SCULLARD 1974, p. 126-133; 146-149. La fondation de Ptolémaïs dite « des chasses », première base de chasse à être fondée sur ordre de Ptolémée II, a été effectuée entre 270/269 et 265/264 a. C. (Thiers 2007, p. 11).

[11] Élien, De la nature des animaux, XI 14 (traduction A. Zucker). L'intelligence et la sensibilité de I'éléphant sont admises et admirées dans le monde méditerranéen antique (voir, par exemple, Pline I'Ancien, Histoire naturelle, VIII 1-12).

[12] Athénée de Naucratis, Deipnosophistes, XIII 85, 606f-607a = Phylarque, FGrH 81 F36.
[13] WiLL 2003, p. 220. On sait que Séleucos I avait, à l'extrême fin du IVe siècle, obtenu plusieurs centaines d'éléphants dressés du souverain indien Chandragupta (SCULlaRd 1974, p. 97-98).

[14] Tel Eudamos, qui avait rallié Eumène de Cardia (infra, n. 23).

[15] Il fallut sans doute du temps avant que des non Indiens maîtrisent l'art du dressage : les premiers dresseurs au service de Ptolémée II ne pouvaient être que des Indiens ; on imagine qu'il en fut de même pour Carthage.

[16] Voir Polybe, Histoires, II 56. 


\section{DES INDIENS ET LEURS FAMILLES DANS LE MONDE MÉDITERRANÉEN À L'ÉPOQUE DES DIADOQUES}

Les armées royales hellénistiques, on le sait bien, avaient une dimension multi-ethnique. La rencontre des Méditerranéens et des peuples « périphériques » permit des contacts et des phénomènes d'acculturation [17]. Si I'on prête avec raison beaucoup d'attention aux Galates ou aux Thraces, il ne faut pas négliger la présence des Indiens, sans lesquels les bataillons d'éléphants, cette arme spectaculaire des armées royales, du temps des Diadoques jusqu'à la guerre antiochique, n'auraient pas fonctionné.

Combien donc furent-ils à émigrer, eux et leurs nomima, dans le monde méditerranéen ? Il y avait dans les armées des Diadoques, en fonction des effectifs en éléphants, de plusieurs dizaines à plusieurs centaines d'experts indiens. On peut, en effet, le déduire du décompte, nécessairement incomplet, des éléphants indiens qui avaient circulé dans la seule Europe jusqu'à l'époque du siège de Mégareon ne tient compte ni de l'Égypte, ni de l'ensemble Syrie-Palestine-Phénicie, où se trouvaient une partie des très nombreux éléphants séleucides. Les éventuels renouvellements du cheptel nous sont inconnus :

- Soixante-dix éléphants ramenés en Europe par Antipatros après la rencontre de Treiparadisos (321 a. C.). Ces éléphants passent entre les mains de Polyperchon après la mort d'Antipatros (319 a. C.) [18].

- Une partie des éléphants - le nombre est indéterminé - de Polyperchon passent entre les mains de Cassandros. À la même époque une partie des éléphants de Polyperchon meurent de faim au siège de Pydna (316 a. C.) [19].

- En 294 a. C., Démétrios s'empare de la Macédoine (Cassandros est mort en 297) et récupère ce qui reste du troupeau de son rival. Il est possible qu'il ait amené un certain nombre de ceux qui lui restaient après la bataille d'Ipsos [20].

- En 280 a. C., avant de partir en Italie, Pyrrhos a, selon les sources, vingt éléphants (pris à Démétrios en 288, quand celui-ci a été expulsé de Macédoine par Lysimaque et Pyrrhos ?) ou cinquante éléphants (prêtés par Ptolémée Keraunos, qui s'empare de la Macédoine en 280) [21]. Mais la confusion des événements (conflit entre Lysimaque et Pyrrhos, entre Lysimaque et Séleucos) ne permet pas de retracer l'origine des éléphants qui circulent en Europe en 280.

- En 274 a. C. Pyrrhos revient en Grèce pour reprendre la Macédoine. Gonatas est vaincu et perd ses - vingt ? - éléphants, hérités de Cassandros. Au siège de Sparte, Pyrrhos déploie vingt-quatre éléphants [22]. En 272, Antigonos Gonatas défait Pyrrhos. Il récupère ses animaux - plus de vingt ? - Une partie de ces éléphants ont été déployés contre Mégare.

Nombreux furent donc les soldats originaires des différentes parties du monde grec qui, de près ou de loin, côtoyèrent des Indiens, leur langue et leur mœurs. À cet égard, il ne fait aucun doute que I'histoire mémorable, « paradoxale » au sens antique du terme, de Nikaia avait fait le tour du camp d'Antigonos et s'était diffusée assez largement pour que Phylarque puisse la recueillir. Il dut y avoir bien d'autres « anecdotes indiennes », toutes perdues sauf une. Celle-ci dut également être assez remarquable pour avoir été admise dans I'historiographie. Elle nous vient de Diodore de Sicile - en réalité, de Hiéronymos de Cardia - et vaut la peine d'être largement citée. Les faits se déroulèrent dans le campement d'Eumène de Cardia, en 318 a. C., après la bataille de Parétacène. Un «stratège » indien, le chef d'un bataillon indien venu avec Eudamos [23]-il n'est pas explicitement qualifié de chef d'un batailIon d'éléphants - , était mort. Ce qui suit repose sur, de toute évidence, un témoignage visuel :

« Keteus, le stratège des soldats venus de l'Inde (ó

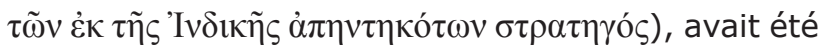
tué dans la bataille, après avoir brillamment combattu. Il laissait deux femmes qui l'avaient accompagné à l'armée. L'une était jeune mariée, I'autre l'avait épousé quelques années auparavant, et toutes deux l'aimaient tendrement [suit une digression sur l'origine du rite nommé « sati »[24]]. La loi ordonnait qu'une seule femme fût brûlée avec le mort, mais elles étaient toutes deux présentes pour les funérailles de Keteus, se disputant le droit de mourir avec lui, comme s'il s'agissait d'une récompense suprême. Devant les stratèges qui faisaient

[17] Cette question fait l'objet de l'ouvrage de CouvenHES \& alii 2011.

[18] SCULLARD 1974, p. 81.

[19] SCULLARD 1974, p. 84.

[20] SCULLARD 1974, p. 99-100 (effectif non mentionné).

[21] SCULLARD 1974, p. 100.

[22] SCULLARD 1974, p. 117-118.

[23] Eudamos gouvernait une satrapie couvrant une bonne partie du Pendjab pakistanais (voir BERNARD 1985, p. 83-85).

[24] Rite imposant à la veuve de se suicider sur le bûcher funéraire de son époux. 
office d'arbitre, la plus jeune déclara que l'autre était enceinte et ne pouvait invoquer la loi. Mais, selon I'aînée, la justice voulait que celle qui l'emportait par les années le fît aussi par les honneurs : ne voit-on pas, dans tous les autres domaines, les aînés être plus respectés et plus honorés que les plus jeunes ? Les stratèges, auxquels ceux qui s'y connaissaient en obstétrique avaient confirmé la grossesse de l'aînée, donnèrent gain de cause à la plus jeune. À la suite de quoi, celle qui avait perdu sa cause s'en alla en sanglotant, après avoir arraché le diadème qui ceignait sa tête et en s'arrachant les cheveux, comme à l'annonce d'un grand malheur, tandis que l'autre, ravie de sa victoire, s'en allait vers le bûcher, couronnée de bandelettes par les femmes de sa maison et magnifiquement parée, comme si sa famille la conduisait à une cérémonie nuptiale, en chantant un hymne en I'honneur de sa force d'âme. Quand elle fut près du bûcher, elle retira sa parure et la distribua à ses parents et à ses amis pour, en quelque sorte, laisser un souvenir d'elle à ceux qui I'aimaient. Voici en quoi consistait sa parure : à ses doigts, une grande quantité de bagues garnies de pierres précieuses de couleur variée ; sur sa tête, de très nombreux ornements d'or en forme d'étoile mêlés de pierres de toute sorte. À son cou une grande quantité de colliers, dont le diamètre, très petit au départ, augmentait progressivement. Enfin, quand elle eut embrassé ses proches, son frère la fit monter sur le bûcher et elle acheva héroïquement sa vie, objet d'admiration pour la foule assemblée pour le spectacle. Toute l'armée en armes [25] fit trois fois le tour du bûcher avant qu'on I'alluma ; quant à elle, une fois étendue auprès de son mari, elle ne laissa échapper, durant la progression rapide du feu, aucune plainte et inspira ainsi, à certains spectateurs de la pitié, aux autres une admiration extraordinaire. Cependant certains des Grecs blâmaient ces usages comme sauvages et cruels. [26]»

Il faut, comme il convient, tenir compte des contraintes et effets de la composition littéraire. Le récit de Hiéronymos de Cardia porte sa part d'interpretatio Graeca, notamment dans I'explication des origines de la «sati ». Il y a également des allusions évidentes, destinées à un public qui connaissait les grands moments de l'expédition d'Alexandre : le bûcher de l'épouse, au courage duquel l'armée rend honneur, fait évidemment écho au suicide du brahmane Kalanos, lui aussi honoré par l'armée du roi, éléphants compris [27]. Restent toutefois des éléments factuels spécifiques à cet événement : les épouses - ou, tout au moins, I'une des deux - étaient accompagnée de sa famille ( $\tau \tilde{\omega} v$

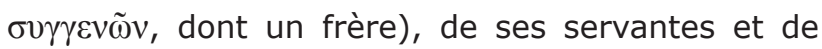

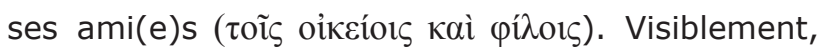
toute la maison de Keteus -évidemment un homme d'un rang supérieur - s'était déplacée dans le camp grec. Quant à l'armée d'Eumène, elle fut le témoin direct d'une série de nomima indiens : chants, habits et ornements, bûcher et cérémonie funèbre et, bien entendu, le spectaculaire suicide de la plus jeune veuve. Enfin, fait rare dans I'historiographie, place est faite aux réactions et émotions des hommes de la «troupe »-ceux-là mêmes qui avaient honoré la jeune femme pour sa vertu pour

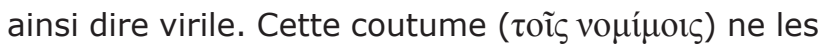
avait pas laissés indifférents : à côté des admirateurs du courage de l'épouse, ceux qui avaient été les plus émus y voyaient quelque chose d'excessif : c'est ce qu'exprime leur jugement sur les mœurs

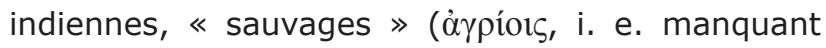
d'hemerôtes) et insupportables ( $\chi \alpha \lambda \varepsilon \pi$ oĩ $\varsigma$ ). De façon assez similaire, une partie de I'armée gréco-macédonienne avait été heurtée par la « sauvagerie » des rites funéraires des habitants de Bactres (Balkh) : suivant une pratique funéraire de type iranien, ils donnaient les individus atteints de maladies incurables - et donc encore vivants - en pâture à des chiens, à l'intérieur même de la ville [28]. Quoi qu'il en soit, tout comme I'histoire de l'éléphante Nikaia, le suicide de la veuve de Keteus marqua les esprits de milliers de témoins avant de gagner sa dignité littéraire. Et I'on peut penser que des scènes du même genre, quoique moins spectaculaires, ne furent pas uniques. Les pertes, en effet, furent nombreuses, parmi les cornacs.

\section{LES ARMÉES HELLÉNISTIQUES ET LA FORMATION DE L'IMAGE DE L'INDE}

Comme l'a écrit Chr. Jacob, l'expédition d'Alexandre en Inde avait profondément modifié I'image traditionnelle de cette contrée, jusqu'alors principalement modelée, pour ce qu'il nous est donné d'en savoir, par Hérodote et Ctésias. L'afflux de nouveaux

[25] Plus de 40000 hommes avant la bataille de Parétacène.

[26] Diodore de Sicile, XIX 33-34 (traduction de P. Goukowsky).

[27] Voir, entre autres témoignages, Strabon, XV 1, 63-65; 68 ; Plutarque, Vie d'Alexandre, 65 ; Arrien, Anabase d'Alexandre, VII 2, 3-4.

[28] Strabon, XI 11, 3 = Onésicrite, FGrH 134 F 5. 
renseignements, issus d'informations et d'observations de première main - principalement dues aux compagnons d'Alexandre et à Mégasthène - avait permis, d'Ératosthène à Strabon, un travail de sélection critique des données relatives à la topographie, à l'ethnographie ainsi qu'à la description des lieux, des animaux et des plantes. Les relations de voyages, particulièrement dans les pays des confins de l'oikoumenê, étant susceptibles de céder à l'invention, il ne fallait garder que ce qui était, autant que possible, débarrassé du pseudos et du muthos [29]. En conclusion, Chr. Jacob souligne à quel point le savoir sur l'Inde ressortit à l'écrit et à la tradition littéraire :

«Contrairement à Thulé, connue par une source unique, I'Inde a donné lieu à une tradition littéraire continue, qui impose sa propre logique, ses thèmes récurrents, ses stéréotypes et son imagerie, même aux auteurs qui peuvent en avoir une connaissance de première main. L'Inde des géographes hellénistiques est avant tout une bibliothèque qui se perpétue et prolifère $(\ldots)[30] \gg$.

Pourtant, en réfléchissant au suicide de la veuve de Keteus et à l'amour maternel de l'éléphante Nikaia, I'historien se prend à imaginer qu'une certaine image de I'Inde a pu se former non dans les bibliothèques, mais ailleurs, et en particulier au sein des armées hellénistiques. Des milliers d'hommes, y compris en Europe dès 321 a. C., vécurent au contact des bataillons d'éléphants et de leurs cornacs indiens. Toutes sortes de nomima indiens s'étaient introduits dans la vie du camp. Ces soldats, témoins de scènes parfois étonnantes - et grandement différentes des usages grecs, comme l'écrit Diodore de Sicile

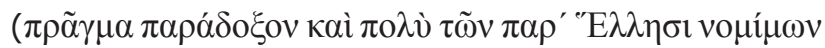
$\dot{\varepsilon} \xi \eta \lambda \lambda \alpha \gamma \mu \varepsilon ́$ vov [31]) -, n'auraient-ils pas pu, eux aussi, contribuer à la formation d'une certaine image de l'Inde ? Ce n'est sans doute pas le fait du hasard si la «matière indienne » que ces deux anecdotes

[29] ]АСОВ 1995, p. 72.

[30] JACOB 1995, p. 80 (voir aussi DiHLE 1964).

[31] Diodore de Sicile, XIX 32, 3 (cette formule introduit I'épisode du suicide de la veuve de Keteus).

[32] Voir, par exemple, Aristote, H.A., IX 25, 620b11 (ce qui est communément rapporté au sujet de la

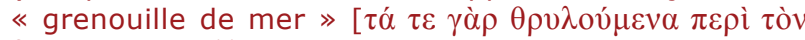

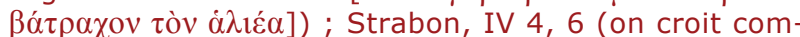
munément que les Gaulois sont portés au plaisir [ $\tau 0 \tilde{\tau} \tau 0$

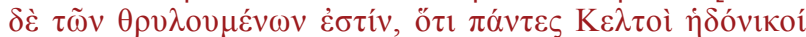

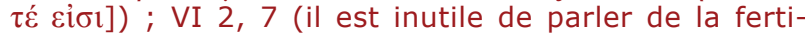

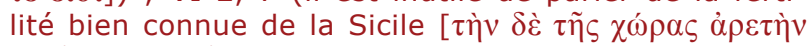

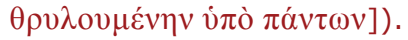

[33] Strabon, XV 1, 30 = Onésicrite, FGrH 134 F21 ; voir aussi Strabon, XV 1, 62 = Aristobule, FGrH 139 F 42. contiennent était vouée à devenir largement connue et à faire partie des lieux communs indiens que I'on oserait presque qualifier de «populaires » - I'un des termes grecs qui renvoie à cette notion de savoir lar-

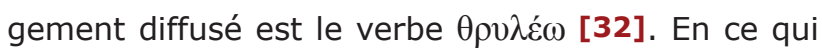
concerne la « sati », la scène observée par l'armée $d$ 'Eumène venait confirmer, à une échelle plus large, ce qu'Onésicrite avait rapporté au sujet du seul peuple indien des Kathaioi [33]. S'ajoutant à I'histoire, elle aussi devenue populaire, de Kalanos [34], le suicide des épouses façonnait l'image d'un pays où I'on ne craignait pas la mort volontaire [35]. Et, en tout état de cause, la « sati » fait partie des lieux communs relatifs à I'Inde [36]. Quant à l'idée que les éléphants comprennent naturellement la langue indienne - présente dans le texte d'Élien-, nul doute que les soldats qui avaient pendant de nombreuses années côtoyé les cornacs avaient contribué à la répandre largement. De la diffusion de ce trait indien, nous avons une preuve indirecte par Élien : lorsque Ptolémée II fit élever un éléphanteau - africain - en lui faisant donner des ordres en grec, il

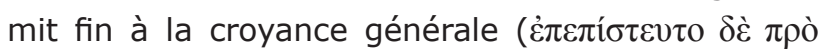

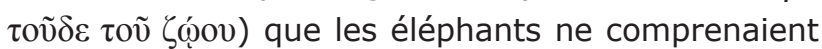
pas d'autre langue que la langue indienne $(\tau \tilde{\eta} \varsigma$ 'I $v \delta \tilde{\omega} v$

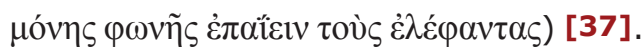

\section{CONCLUSION}

Le suicide des veuves, ou la relation subtile entre les Indiens et leurs éléphants domestiques peuvent sans hésitation être classés dans la catégorie des

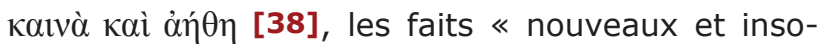
lites » observés en Inde par les Gréco-Macédoniens. On est, d'une certaine manière, dans le domaine des paradoxa qui ont presque systématiquement fait partie des descriptions « classiques » de I'Inde, comme le note Chr. Jacob :

[34] Voir SCHNEIDER 2008, p. 253-259, au sujet d'un papyrus de Zénon. Pour citer un autre exemple, Éléazar, cherchant au cours de la révolte juive de 66-70 p. C., à ranimer le courage de ses partisans - hommes que I'on n'imagine pas tous frottés aux lettres grecques -, leur rappelle que les sages indiens montent sur le bûcher sans trembler (Flavius Josèphe, Guerre des Juifs, VII 351-357).

[35] Voir, Cicéron, Tusculanes, V 77-78.

[36] Voir, par exemple, Stobée, CXXIII12 = Nicolas de Damas, fr. Müller 143 ; Properce, III 13, 15 ; Plutarque, Mor., 499c.

[37] Élien, De la nature des animaux, XI 25.

[38] Strabon, XV 1, 62 = Aristobule, FGrH $139 \mathrm{~F} 42$ (à propos des nomima observés à Taxila). 
« Les merveilles de I'Inde, rapportées par Mégasthène et Déimaque, ont fourni nombre de matériaux à un courant littéraire de l'époque hellénistique, la paradoxographie, qui se développe comme un genre autonome à partir de Callimaque (...). Recueil de merveilles du monde naturel (fleuves, pierres, plantes, animaux) ou de curiosités anthropologiques (particularités physiques et coutumes des peuples), le texte paradoxographique générait auprès de ses lecteurs un plaisir spécifique, où se mêlent le littéraire et l'esthétique, la curiosité parascientifique (si tant est que cet adjectif ait un sens dans I'Antiquité), et les motivations passionnelles et fantasmatiques [39] ».

Nul doute que cette «paradoxographie » ne doive son existence et son succès aux travaux des lettrés.
Mais elle n'est pas sortie seulement des bibliothèques et des livres. La guerre et les institutions militaires ont joué leur rôle : des milliers d'anonymes avaient rapporté et diffusé ce qu'ils avaient vu ou entendu. Telle fut leur discrète contribution à la formation des lieux communs relatifs à I'Inde, dont Lucien recommande ironiquement de faire parfois usage dans l'art oratoire [40].

\section{[39] ]АСОВ 1995, p. 73.}

[40] Lucien, Le maître de rhétorique, 18, recommandant d'évoquer les coutumes de I'Inde et d'Ecbatane si le sujet concerne un homme violent ou adultère à

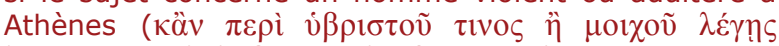

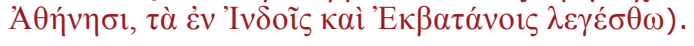

\section{BIBLIOGRAPHIE}

Bernard, Paul, 1985, «Le monnayage d'Eudamos, satrape grec du Pandjab et 'Maître des éléphants' », in Gherardo Gnoli \& Lionello Lanciotti (éd.), Orientalia losephi Tucci Memoriae Dicata, Roma, p. 65-94.

Bongard-Levin, Grigorij Maksimovič, 1985-1986, «Ancient India and the Graeco-Roman World », Indologica Taurinensia. The Journal of the International Association of Sanskrit Studies, 23 (Proceedings of the "Sixth World Sanskrit Conference", Philadelphia, October $\left.13^{\text {th }}-20^{\text {th }}, 1984\right)$, p. 168-185.

Couvenhes, Jean-Christophe, Crouzet, Sandrine, PéRé-Nogues, Sandra (éd.), 2011, Pratiques et identités culturelles des armées hellénistiques du monde méditerranéen (Hellenistic Warfare 3). Actes du colloque tenu à Tours, les 23 et 24 mars 2007, Bordeaux.

DiHLE, Albrecht, 1964, « The conception of India in Hellenistic and Roman literature », Proceedings of the Cambridge Philological Society (New Series), 10, p. 15-23.

Doshi, Saryu (éd.), 1985, India and Greece: Connections and Parallels, Bombay.

Filliozat, Jean, 1956, Les relations extérieures de I'Inde (I) [Les échanges de l'Inde et de l'Empire Romain aux premiers siècles de l'ère chrétienne. La doctrine brahmanique à Rome au III siècle], Pondichéry.

Hammond, Nicholas G. L., 1988, A History of Macedonia. Volume III: 336-167 B.C., Oxford.

Heinen, Heinz, 1972, Untersuchungen zur hellenistischen Geschichte des 3. Jahrhunderts v. Chr. : zur Geschichte der Zeit des Ptolemaios Keraunos und zum chremonideischen Krieg, Wiesbaden.

ЈАСов, Christian, 1995, «L'Inde imaginaire des géographes alexandrins », in Jean-Claude Carrière, Évelyne Geny, Marie-Madeleine Mactoux, François Paul-Lévy (éd.), Inde, Grèce ancienne : regards croisés en anthropologie de l'espace, Paris, p. 61-80.

Le Bas, Philippe, 1842, Recherches historiques et critiques sur cette question : à qui doit être attribué le siège de Mégare dont parlent Polyen et Élien ?, Paris.

Muckensturm-Poulle, Claire \& Geny, Évelyne, (éd.), 2010, Inde-Grèce : regards et influences, Besançon (Dialogues d'histoire ancienne, Supplém. 3).

Rawlinson, Hugh G., 1926, Intercourse between India in the Western World from the Earliest Times to the Fall of Rome, Cambridge.

Sedlar, Jean W., 1980, India and the Greek World. A Study in Transmission of Culture, Totowa.

SChNeIDer, Pierre, 2008, «Res Indicae : quelques remarques au sujet du papyrus PCZ. 59532 et du fragment Sat. 66V² d’Ennius », in José María Candau Morón, Francisco José González Ponce, Antonio Luis Chávez Reino (éd.), Libyae lustrare extrema. Estudios en honor del Profesor Jehan Desanges, Sevilla, p. 253-270.

Scullard, Howard H., 1974, The Elephants in the Greek and Roman World, London.

TARN, William W., 1913, Antigonus Gonatas, Oxford.

ThIers, Christophe, 2007, Ptolémée Philadelphe et les prêtres d'Atoum de Tjékou. Nouvelle édition commentée de la « stèle de Pithom » (CGC 22183), Montpellier.

Treloar, Alan, 1977, « Ancient Greece and India », Prudentia 9, p. 81-96.

WILL, Édouard, 2003, Histoire politique du monde hellénistique : 323-30 av. J. -C, Paris. 